\title{
El rol "accesible" del espacio público
}

\author{
Maysel Castillo García
}

Artículo

\section{Resumen:}

Invitada internacional

Institución: Universidad de Barcelona. España

E-mail: mayselcastillo1983@gmail.com

Recibido: 1 de junio de 2018

Aprobado: 6 de diciembre de 2018

\section{Maysel Castillo García}

Arquitecta por el Instituto Superior Politécnico "José Antonio Echeverría" en La Habana. Master en Diseño Urbano: Arte, Ciudad y Sociedad por la Universidad de Barcelona. Doctoranda de la Línea de Investigación "Sociedad, Cultura y Territorio" del programa de Doctorado "Geografía, Planificación Territorial y Gestión Ambiental" en la Universidad de Barcelona.
Uno de los principales factores que condiciona la vida cotidiana de un barrio o ciudad, lo constituye la accesibilidad. La accesibilidad depende de muchos factores como el diseño, los usos, entre otros, pero principalmente de la complejidad de las relaciones ciudadanas en el espacio urbano; por tanto, hablar de accesibilidad urbana, es hablar de urbanización y aún más, de ciudad como producto para la sociedad. La accesibilidad al medio físico debe ser considerada como un derecho de todo ser humano a tener la posibilidad de ingresar, transitar y permanecer en un lugar, de manera segura, confortable y autónoma. La calidad de vida de los ciudadanos está condicionada por la calidad del espacio público urbano donde habitualmente desarrollan sus actividades, donde se crean posibilidades de encuentro, de relaciones sociales y de contacto con la naturaleza. Los análisis llevados a cabo para evaluar el espacio público con una perspectiva accesible deben partir de un enfoque sistémico que analice los problemas del espacio público en su conjunto y no de los componentes aislados. Hoy se trata de considerar y organizar estas relaciones con base al adecuado funcionamiento de todas las personas en el espacio público desde las diversas redes que hacen accesible al territorio. La reflexión que plantea este artículo; forma parte de los resultados teóricosmetodológicos de la tesis doctoral "La ciudad como producto social. Construcción de espacio público accesible", dentro del programa de doctorado Geografía, Planificación Territorial y Gestión Ambiental, de la Facultad de Geografía e Historia de la Universidad de Barcelona.

Palabras clave: ciudad; espacio público; accesibilidad universal; movilidad urbana.

\section{The "accessible" role of public space}

\begin{abstract}
:
One of the main factors that conditions the daily life of a neighbourhood or city is accessibility. The accessibility depends on many factors such as design or uses, but mainly on the complexity of the citizens' relationships in the urban space. Therefore, to talk about urban accessibility is to talk about urbanization and even more, about the city as a social product. Accessibility has to be considered as a human right to have the possibility of entering, transiting and staying in a place, in a safe, comfortable and autonomous way. Citizens' quality of life is conditioned by the quality of the urban public space where they usually develop their activities. The analyses carried out to evaluate the public space with an accessible perspective should be based on a systemic approach that analyses the problems of the public space as a whole and not of the isolated components. Today it is about considering and organizing these relationships based on the adequate functioning of the public space from the various networks that make accessible the urban Territory This article reflects on the theoric and methodologic results of the doctoral thesis: "The City as a social product. Construction of accessible public space", within the doctorate program Geography, Territorial Planning and Environmental Management at the Faculty of Geography and History of the University of Barcelona.
\end{abstract}




\section{Introducción}

La ciudad se define hoy como una realidad "difusa, diversa, mutante, global, compleja, incierta", donde los problemas urbanos se presentan tan complejos que requieren respuestas contundentes y a la vez flexibles, que puedan garantizar soluciones desde la perspectiva de los ciudadanos (Brandao, 2011). Las características de las sociedades del siglo XXI definen nuevos retos para el urbanismo contemporáneo, tanto en los procesos de transformación urbana de las ciudades consolidadas, como en la construcción del nuevo urbanismo. Hoy la ciudad acumula problemáticas sin respuestas adecuadas a los tiempos y acordes a las nuevas fuerzas sociales.

Se reclaman nuevas formas de hacer ciudad, un urbanismo más cercano a sus ciudadanos, un nuevo urbanismo que no solo sea producto del ejercicio de técnicos y políticos; sino uno en el que exista mayor participación de la población en las cuestiones que tienen que ver con el desarrollo y la vida colectiva (Capel, 2005). Este nuevo urbanismo debe tener necesariamente que ver con un cambio de mentalidad para producir una ciudad; en donde esta sea el resultado del concierto entre el ciudadano, la visión del desarrollo y el conjunto de políticas públicas que se generan para ello.

Un nuevo urbanismo que traduzca la ciudad concebida por y para sus ciudadanos, en hechos urbanos capaces de transformar y mejorar la calidad de vida de sus habitantes. Un urbanismo en el que la ciudad percibida sea la representación de una visión de calidad de vida, progreso y desarrollo, en el que exista una relación entre el ciudadano como individuo y como elemento de un engranaje colectivo y finalmente, un urbanismo, en el que la ciudad vivida se asuma como propia, en la que los ciudadanos se identifiquen, no solo porque representa lo que son y desean, sino porque les permite la realización como sujetos individuales y colectivos (Lefevre, 1974).

La teoría urbana, sitúa el desarrollo de las ciudades bajo dos perspectivas relacionadas entre sí: la morfología de las ciudades y la forma de relacionarse del ciudadano con el espacio y el entorno en el que desenvuelve su vida pública. Una parte importante de dicha base teórica tiene como objeto de estudio la ciudad que surge a partir del proceso de industrialización experimentado durante el siglo XIX, contexto en el que emergen gran parte de los paradigmáticos planes de reforma y crecimiento de las ciudades, que a su vez han dado soporte a la creación de modelos de planificación urbana posteriores.

Los procesos de evolución y transformación urbana que han experimentado algunas ciudades en el siglo XIX; han servido de escuela en la planeación de la ciudad actual. En las últimas décadas se ha presenciado una transformación gradual de las ciudades y de los espacios de nuestra cotidianidad. Gran parte de estos procesos se han generado por la puesta en marcha de una serie de planes, programas y proyectos de diversa naturaleza como resultado de fenómenos económicos, culturales y tecnológicos. La sociedad urbana se ha hecho más compleja, modificando en este proceso; los comportamientos sociales, el uso del tiempo y las formas de acceso y comunicación.

La ciudad del nuevo milenio produce nuevas formas de vivir, construir y transformar los espacios físicos territoriales y se enfrenta a serias contradicciones y nuevas paradojas. En una coyuntura nacional donde surgen desigualdades con las que tendremos que aprender a convivir, el espacio público puede ayudar a suavizarlas al hacer coincidir sobre un territorio muy compacto y significativo a personas con niveles de ingresos y condiciones de vida diferentes (Coyula, 2000).

El espacio público es una posible respuesta al difícil y novedoso desafío de articular desde un conjunto urbano más o menos homogéneo, el barrio, la ciudad aglomerada, hasta una región metropolitana. La continuidad de los grandes ejes de espacio público es una condición de visibilidad y de accesibilidad para cada uno de los fragmentos urbanos y un factor esencial de integración ciudadana (Borja, 1998), lo que promueve la articulación urbana.

La ciudad ha sido siempre un espacio de convivencia, pues su continuidad y conectividad condiciona las relaciones que en ella se producen. No resulta extraño pensar que caminar es la actividad física natural que utilizamos por excelencia y de alta relación con las actividades cotidianas como el comercio, el trabajo, la cultura o el tiempo libre. La aparición del vehículo supuso una nueva forma de desplazamiento y por ende un 
cambio radical en la fisonomía urbana de la ciudad y en las formas de relacionarse con esta. Retomar "el caminar" en la vida urbana ha sido un reto importante de los últimos 50 años. Se ha procurado un gran esfuerzo en hacer atractiva la ciudad para invitar al individuo a pasear 0 desarrollar sus actividades cotidianas, sin la dependencia del vehículo.

Para afrontar el desafío que significa el derecho a la accesibilidad de todas las personas como necesidad de conexión social, muchas ciudades han concebido instrumentos de programación de medidas que se conocen como los "planes de desplazamientos" o de "movilidad urbana". Esta reflexión contempla la necesidad de un modelo de movilidad que conlleve a un tipo de ciudad más sostenible, entendiéndose por ello un espacio urbano donde se minimice el consumo de energía y la contaminación producida y se maximice el intercambio de información (Herce, 2000). Se trata de plantear un modelo que priorice el viaje a pie, en bicicleta o en transporte público con el objetivo de disminuir los consumos energéticos, pero también de resolver las necesidades de conexión social.

Para alcanzar un modelo de ciudad sostenible; es preciso articular aspectos importantes a distintas escalas de planificación territorial: la distribución de los usos de suelo y la oferta de los servicios de transporte que garanticen las relaciones entre las actividades, por ello el plan de movilidad urbana es ante todo un instrumento para la reflexión sobre la ordenación territorial y sobre el crecimiento de la ciudad. Indiscutiblemente la creación de nuevos servicios, definirá el número de desplazamientos de una ciudad, que dependen de muchos factores pero fundamentalmente de la complejidad de las relaciones humanas que aumentan y cambian los procesos económicos, sociales y el acceso a la información por parte de los ciudadanos.

Es de vital importancia manejar conceptos y enfoques de temáticas relacionadas con el uso del espacio público urbano, como punto sensible para actuar en la ciudad. Algunos de estos enfoques están dedicados a las movilidades que se generan en la ciudad, los niveles de acceso y la accesibilidad para todos, que se retoman como problemática principal de abordaje sobre el espacio público urbano en el marco de este artículo.

Abordar la movilidad urbana y la accesibilidad de los espacios públicos demanda profundizar en aspectos no solo físicos asociados al espacio, sino también sociales, económicos y culturales, así como su evolución en la sociedad contemporánea. El fenómeno urbano en conjunto con los retos socio - demográficos en el siglo XXI imponen un diseño que tenga en cuenta la integración de diferentes conceptos para favorecer la inclusión social y responder a los retos de la diversidad del ser humano.

\section{Antecedentes}

"Desde una perspectiva histórica el espacio en las ciudades ha cumplido tres funciones vitales a lo largo del tiempo: lugar de encuentro, mercado, y espacio de conexión. Como lugar de encuentro la ciudad era escenario donde ocurría el intercambio de información social de todos tipos. Como mercado, los espacios abiertos de la ciudad eran el territorio donde se llevaba a cabo el intercambio de bienes y servicios. $Y$ finalmente las calles de la ciudad proveían de acceso y conexiones entre todas las funciones de la ciudad" (Gehl; Gemzoe, 2001, p. 90).

Este patrón de uso de la ciudad histórica es verificable en todas las etapas del desarrollo de los asentamientos urbanos. Desde las ciudades de la antigua Grecia, las ciudades romanas, medievales, renacentistas y barrocas, hasta las ciudades de la era de la ilustración y la revolución industrial, el espacio urbano ha estado siempre desbordante de actividad humana y funciones a través de la historia. La vida en las ciudades era parte integrante y esencial de la sociedad.

El movimiento Moderno a partir de mediados de la década de los 20, en su búsqueda por proveer a la creciente población urbana de ciudades más limpias y saludables, degradó dramáticamente la importancia de los espacios públicos tradicionales. Las calles y las plazas fueron declaradas poco saludables y las actividades en dichos lugares fueron severamente criticadas y tachadas de ser poco beneficiosas para la sociedad. 
El libro "Muerte y Vida de las grandes ciudades americanas", publicado por Jane Jacobs en 1961 marcó un punto de viraje en la erosión gradual del concepto de espacios públicos y vida pública urbana. A partir de este momento comenzó a desarrollarse una serie de eventos que demostraban el proceso de revaloración del espacio público urbano (el cierre de ciertas calles al tráfico, la introducción de calles peatonales, así como un gran número de publicaciones promoviendo el concepto de espacio y vida pública). Esta orientación del diseño y planeamiento urbano hacia una jerarquización de la vida y el espacio público ha perdurado alrededor de 50 años incrementando su intensidad a lo largo del tiempo.

La transformación de las ciudades como resultado de su crecimiento ha fragmentado el territorio. La aparición de diversas formas de ocupación ligadas a actividades que no tienen dependencia de los núcleos urbanos genera territorios donde se intercalan usos y se mezclan espacios centrales y periféricos; donde existen actividades cambiantes y dependencias de funciones a determinadas distancias. La dinámica de usos y recorridos genera movilidades, y las infraestructuras siguen siendo uno de los soportes más importantes del desarrollo económico, ya que su papel ha llevado a la reivindicación de redes y al aumento de su complejidad.

Las ideas sobre el espacio urbano han variado con el desarrollo de un mejor entendimiento del papel de los mismos en los procesos sociales. En la década de los 50 el urbanismo racionalista manejaba conceptos de segregación funcional que generaron modelos urbanos de escaso valor y de reducida eficiencia. Estos modelos quedaron obsoletos y fueron sustituidos por una reafirmación de los valores de la urbe tradicional.

Con esta vuelta a la ciudad compacta, el espacio público cobra un sentido diferente, planteando nuevos retos vinculados a las accesibilidades, las movilidades, a las centralidades, a las simbologías existentes y a las relaciones sociales que le dan sustento, a su capacidad de inclusión e integración social.

En un contexto donde la vida urbana moderna se desarrolla a través de un conjunto de nodos que se articulan gracias a la capacidad de integrar las formas de desplazamientos y usos, el espacio público se convierte en el gran escenario donde se discuten las posibilidades o limitaciones de una comprensión de ciudad accesible a la población en su conjunto. Se evidencia la necesidad de un espacio público ya sea la plaza, la calle 0 algún otro cuyo diseño propicie la pluralidad de actividades y concurrencia. Esta dimensión demanda un análisis de los actores que dan vida al espacio público. Sobre ello es importante tomar en cuenta la premisa planteada por Borja: "(...) El espacio público concebido también como instrumento (...) de cohesión urbana, de autoestima colectiva (...) de formación y expresión de voluntades colectivas, el espacio de la representación pero también del conflicto." (...) (Borja 2003, p 29). No debe suponerse que son las formas arquitectónicas las que configuran este espacio, pues este constituye sobretodo una construcción social. Por tanto, su configuración parte de las iniciativas de apropiación espacial de la gente que lo usa.

Hay una gran diversidad de peatones en la ciudad con distintos requerimientos para el uso del espacio viario que dependen de su edad, su capacidad para el movimiento y de la forma de llevarlo a cabo, ya sea solos, en compañía de otras personas, con las manos libres, portando bultos, etc. Diseñar la ciudad, las infraestructuras peatonales; para un peatón "atlético" es una medida inaceptable para el modo mayoritario de movilidad urbana.

Las intervenciones en el espacio público se realizan manejando criterios genéricos de diseño y uso de los espacios urbanos, respondiendo a necesidades elementales de los usuarios. Sin embargo es mucho más complejo las relaciones que se establecen en la ciudad y con ello se transforma el modo de hacer ciudad. Las distintas prácticas urbanas de la ciudad contemporánea, ha conllevado a que se tengan en cuenta de forma integral aspectos que inciden en la producción del espacio público como producto social. ¿Se tienen en cuenta para el diseño del espacio público todas las prácticas urbanas que se generan en la ciudad actual? ¿Garantizan los espacios públicos satisfacer las necesidades de todas las personas? ¿Es el espacio público un espacio para las relaciones sociales? ¿Es el modelo de ciudad actual accesible a todos? 


\section{Papel del espacio público}

Para hablar del papel que juega el espacio público urbano en la sociedad contemporánea hay que comenzar definiendo que entendemos por espacio público. El contenido atribuido al concepto de espacio público suele ser muy general, al extremo de que pierde especificidad, o muy restrictivo dado su marcado carácter especialista, tributario de las concepciones del urbanismo moderno. Es un concepto difuso, indefinido y poco claro, que puede incluir la plaza, el parque, la calle, el centro comercial, el café y el bar, así como la opinión pública o la ciudad, en general. Muchas son las teorías sobre el papel que juega el espacio público dentro de la ciudad contemporánea. Incluso existen definiciones muy abarcadoras que incluyen dentro del concepto a ese espacio virtual generado por la difusión de internet en la sociedad del mundo desarrollado.

"Como dice el filósofo Jürger Habermas, la ciudad es el espacio público donde la sociedad se fotografía, el poder se hace visible y se materializa el simbolismo colectivo". Para Henri Lefebvre, es la sociedad inscrita en el suelo. Para el escritor Julio Cortázar un lugar con mucha gente que interactúa cara a cara. Una concentración de puntos de encuentro", donde "lo primero son las calles y las plazas, los espacios colectivos, y sólo después vendrán los edificios y las vías, que son los espacios circulatorios. Y estas son sólo algunas voces que resaltan el sentido público de la urbe". (Ventura, 2012, p.27)

Sea cual sea el origen del concepto, la tendencia es reforzarle como el lugar, que más allá del ámbito individual y privado, constituye el escenario configurado por la ciudadanía y que, por sus condiciones físicas, espaciales y simbólicas, es el lugar por excelencia para evaluar o verificar la calidad de vida urbana en la ciudad.

El espacio público se considera el eje fundamental en los procesos de desarrollo urbano. Como estructurante de la ciudad, su emergencia depende de muchos elementos relacionados. Una de ellas, las políticas públicas, normalmente dependen del marco que da la planificación estratégica del desarrollo en las múltiples escalas del territorio, pero al mismo tiempo obedecen a reivindicaciones o demandas de la ciudadanía.

Cuando nos referimos al término espacio público urbano hablamos de aquel conjunto de estructuras básicas para la vida en sociedad del hombre, con un ordenamiento territorial que satisfaga sus necesidades primordiales. Las infraestructuras y redes que garantizan el funcionamiento de sus elementos arquitectónicos que van configurando la ciudad. Asi mismo el espacio público, se convierte en un elemento imprescindible para conferir significado social, que le otorga sentido a las cosas a través del uso; de los elementos simbólicos, físicos e intangibles, sumado a las condiciones sociales, culturales, políticas, económicas, entre otras, que se orientan para facilitar la apropiación colectiva, la identificación con el lugar y la socialización de la vida en sociedad.

La ciudad constituye la estructura más compleja en la que este tipo de espacio se manifiesta. "El espacio público se emplea también intensamente para otros usos: vender y comprar, pasear, conversar, jugar, mirar y ser vistos" (Coyula, 2000, p.18).

"Lo público es precisamente un ámbito compartido de experiencias urbanas que se desarrollan en espacios propicios o propiciadores." (Saldarriaga, 2002, p.211), por lo que se hace necesario conocer bien el uso social de los espacios públicos. Este uso dependerá de muchos factores: el diseño, la accesibilidad, la belleza, la monumentalidad, la promoción, el mantenimiento, la diversidad de usuarios posibles y la relación de estos con los componentes del espacio público.

\section{Movilidad "sostenible", accesos, accesibilidad y diseño universal}

Abordar la movilidad urbana y la accesibilidad de los espacios públicos demanda profundizar en aspectos no solo físicos asociados al espacio, sino también sociales, económicos y culturales, así como su evolución en la sociedad contemporánea. El fenómeno urbano de conjunto con los retos socio - demográficos en el siglo XXI imponen un diseño que tenga en cuenta la integración de diferentes conceptos para favorecer la inclusión social y responder a los retos de la diversidad del ser humano. 
Figura 1. Ocupación del espacio público con morfologías urbanas similares. A (izquierda): Centro Histórico de Barcelona B (derecha): Centro Histórico de La Habana. Fuente: propia.
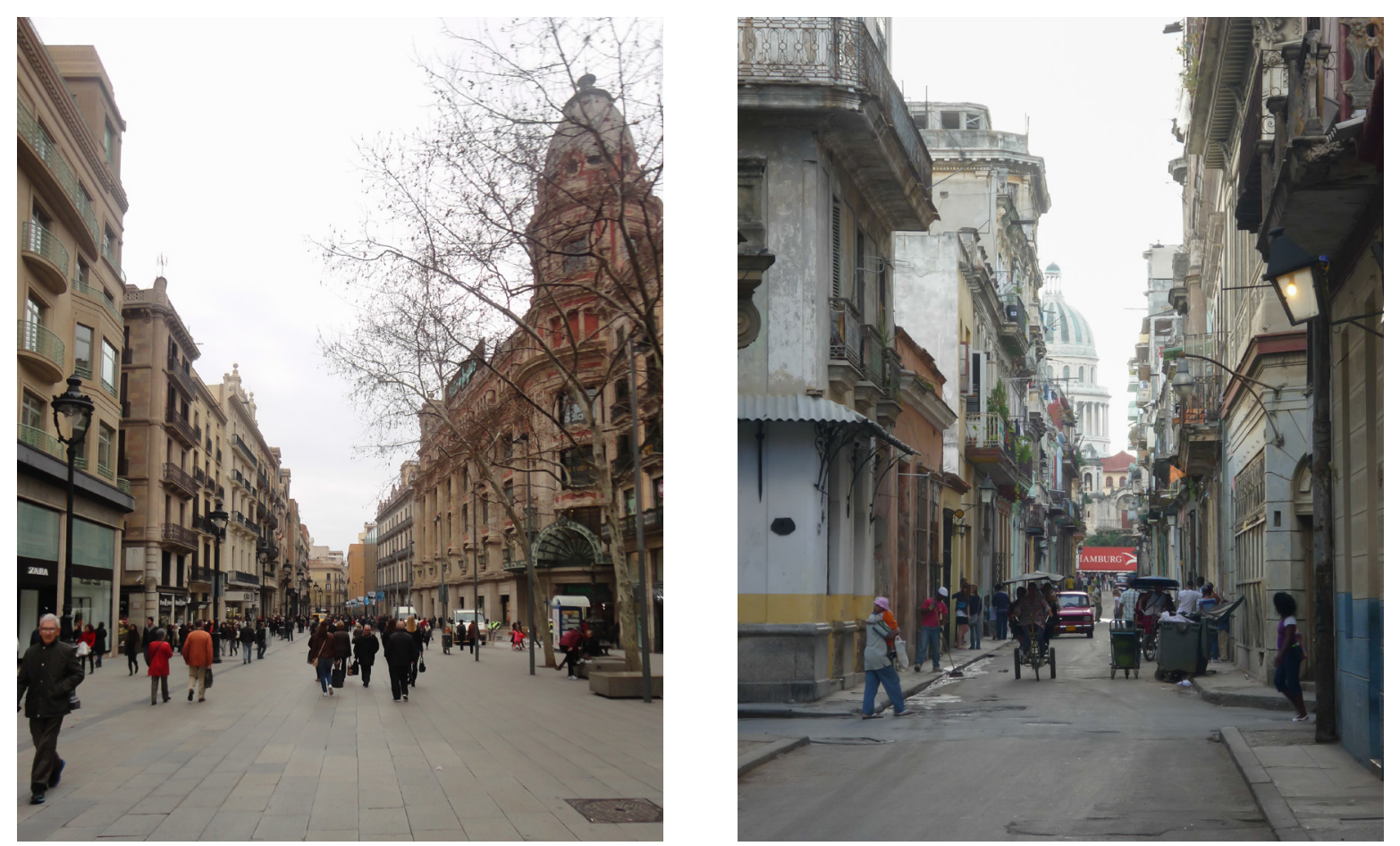

Garantizar el acceso, la comodidad, el bienestar y el desenvolvimiento seguro de todas las personas implica traspasar los límites entre la edificación y el espacio público 0 entre este y el desplazamiento entre un punto de origen y un destino; pues ahí radica la importancia en la continuidad de la cadena de accesibilidad. Es necesario entender la accesibilidad no solo como una condición a cumplir en los espacios, edificaciones y el transporte público de manera aislada, sino como una red articulada necesaria para el desarrollo de la vida cotidiana.

Se trata de entender los conceptos asociados al espacio público accesible a partir de la aplicación sistémica de los principios de la accesibilidad a todos aquellos ámbitos del entorno físico. Considerar diversas visiones e interpretar todos los elementos que garantizan la igualdad de uso, el desenvolvimiento y disfrute en condiciones de seguridad, comodidad, eficacia, autonomía personal, sostenibilidad y uso fácil del espacio público de un modo holístico es lo que favorece la producción de espacio público accesible.

El concepto de accesibilidad se ha ido abriendo camino más allá de la eliminación de barreras físicas, y hoy considera a la persona y a su entorno como un todo. Es por ello que se han desarrollado a partir de él, diversos enfoques cuyo alcance va más allá de las respuestas que ofrecen las normativas. Muchas de ellas han hecho énfasis en las escalas urbanas y del diseño industrial y gráfico. La visión integradora de estas ha sido encaminada a partir del desarrollo del concepto de Diseño Universal, Diseño para Todos o Diseño Inclusivo.

El concepto de accesibilidad al medio físico que se maneja en la actualidad implica no solo el poder llegar y acceder, sino también poder utilizar, tanto los elementos propios $\mathrm{u}$ objetos de los que se ha provisto el medio como sus áreas de uso. El concepto deja explícita la necesidad de que estas acciones sean realizadas con autonomía, seguridad, equidad, salud y bienestar (Cazanave, 2007). La accesibilidad debe ser considerada un bien público, consagrado en términos de derecho ciudadano, a través del cual toda persona, sin importar su edad, género, religión, condición física, tiene derecho a interactuar socialmente y disfrutar, con autonomía, de todos los servicios que proporciona la comunidad. Es posible de esta manera encaminar el logro de una ciudad accesible, eliminando las múltiples barreras que impiden la autonomía personal y la movilidad de los ciudadanos, a fin de mejorar el bienestar social y la solidaridad en la comunidad (CONADIS, 2009).

Según el Diccionario de la Real Academia Española, accesibilidad significa "calidad de accesible", accesible tiene varias acepciones, definiéndose como "que tiene acceso", "de fácil acceso o trato", o de "fácil comprensión, inteligible". El concepto de accesibilidad, que adopta la definición discutida y aprobada por la Organización Mundial de la Salud y las normas internacionales ISO expresa que:

"La cualidad del medio físico cuyas condiciones facilitan acceso, desplazamiento y utilización del mismo de manera autónoma por todas las personas o grupo de personas con independencia de sus capacidades motoras, sensoriales o mentales; garantizando 
salud, bienestar y seguridad durante el curso de las tareas que realiza en dicho medio físico." (OMS, 2001)

En el presente siglo se desarrolla una visión más abarcadora del concepto de accesibilidad al medio físico. Esta dimensión va más allá de la eliminación de barreras físicas, barreras psicológicas y sociales. Se promueve la integración del individuo cualesquiera sean sus capacidades, habilidades y limitaciones en general. El nuevo concepto de accesibilidad aprobado en la CIF y aplicado por organizaciones internacionales; considera un ser imperfecto y diverso, pero con igualdad de derechos para participar de forma activa en el desarrollo de la sociedad contemporánea (OMS, 2001).

A partir de la estrategia de Desarrollo Sostenible que se ha ido desarrollando a nivel mundial; debido al estado de deterioro del ambiente urbano como consecuencia de la contaminación ambiental, surge el concepto de movilidad sostenible. Esta definición tiene como objetivo principal la mejora de la calidad de vida urbana y la aplicación de criterios de sostenibilidad ambiental y social. Un segundo enfoque que se relaciona igualmente con los objetivos del primero lo constituye el de movilidad accesible. Este consiste en la aplicación sistemática; a todos aquellos ámbitos del entorno exterior, de los principios de la accesibilidad para todos, lo que permite el desenvolvimiento, uso y disfrute del mismo, en condiciones de seguridad, comodidad, eficacia, autonomía personal, sostenibilidad y uso fácil. Recientemente conceptos como acceso y accesibilidad son cada vez tomados más en cuenta a la hora de determinar la calidad del diseño de los ambientes construidos. Promover el acceso por medio de la planificación y el diseño es visto como una manera de combatir las fuerzas descentralizadoras de la expansión metropolitana. Algunas formas urbanas poseen inherentemente mejores condiciones de acceso: los patrones de desarrollo de baja densidad necesariamente disminuyen la accesibilidad porque las facilidades se encuentran a mayores distancias y los usos de suelo están segregados.

Esta filosofía tiene como objetivo la construcción de productos, servicios y entornos más sencillos de usar por todas las personas y sin esfuerzo alguno. Queda abolido el concepto de ser humano estándar y se asume una visión abarcadora de lo que significa el ser humano "normal" con capacidades y habilidades diversas. Se intenta resolver el problema con una visión holística, partiendo de la idea de la diversidad humana. Su propósito es simplificar la realización de las tareas cotidianas en beneficio de la población de todas las edades y habilidades. El concepto surge del diseño sin barreras, del diseño accesible y de la tecnología de apoyo, pero a diferencia de estos conceptos el diseño universal alcanza todos los aspectos de la accesibilidad.

La libertad de acceso permite descubrir diversidades sociales y culturales, que nos abre la posibilidad de enriquecer nuestras dimensiones humanas; además, el compartir espacios con lógicas y prácticas distintas nos enseña en concreto a practicar la tolerancia, condición fundamental para poder vivir en democracia. El acceso no está basado solamente en las distancias entre dos locaciones en el espacio, sino que puede también ser influido por factores sociales, barreras culturales o un diseño poco efectivo. Las características sociales, económicas o culturales de los distintos colectivos de población son factores de diferenciación en el acceso y utilización de los bienes.

Las normativas actuales que regulan el desarrollo urbano otorgan a las condiciones de acceso un rol predominante, ya que la accesibilidad tiene un impacto directo en la calidad de vida de los habitantes de cualquier población. El acceso puede ser mejorado por el diseño. Kevin Lynch teorizó sobre esta conexión y proclamó el acceso como un componente clave de su teoría de la forma urbana ideal. En su hipótesis, el acceso es planteado como componente de la calidad sensorial y la legibilidad simbólica del lugar.

Los nuevos urbanistas han desarrollado un método específico de planificación basado en mejorar los niveles de acceso a escala regional (permitiendo una variedad de alternativas de transporte), metropolitana (promoviendo la forma urbana compacta) y de barrios (promoviendo el uso mixto y la densidad habitacional) (Talen, 2001).

Existen cinco tipos de factores que influyen en las formas de medir el acceso y la accesibilidad:

Las dos primeras son sencillamente las locaciones en el espacio del punto de origen y punto de destino, dependiendo sus desplazamientos de los usos de suelo. Usualmente 
el punto de origen se refiere a espacios residenciales y los puntos de destino a entidades que poseen una locación espacial determinada tales como escuelas o centros de trabajo, entre otros usos cotidianos.

Un tercer factor lo constituye la ruta de viaje y su distancia (camino entre origen y destino). Esta incluye no solo la distancia entre dos o más puntos sino las cualidades de la ruta. Los factores que pueden influir en esta incluyen la topografía, la velocidad de circulación, el número de carriles de tráfico, el medio de transporte; y para rutas peatonales la seguridad percibida, la calidad de la acera y los volúmenes de tráfico, así como las diversas características ambientales de las urbes.

Otro factor lo constituyen las características de los individuos que buscan el acceso. Los factores que pueden afectar el acceso en este aspecto incluyen: estatus socioeconómico, edad, género, raza, y presencia de algún tipo de discapacidad.

El factor final que afecta la accesibilidad se encuentra en las características positivas o negativas que presenta el diseño de los espacios de destino y origen referidos a viviendas y servicios a los que necesitan acceder los residentes de las mismas, así como la cantidad, tipo y calidad del servicio ofertado en el destino.

El diseño universal es un enfoque contemporáneo a la hora de enfrentar el proceso de diseño que surge como consecuencia de la necesidad de implementar los nuevos conceptos de igualdad social y de discapacidad desarrollados a finales del siglo XX. Es un concepto complejo que engloba varios aspectos que describen el fenómeno de la interacción entre los individuos y su medio. Podemos hablar de accesibilidad al medio físico, accesibilidad universal, y del acceso como medida de la igualdad social.

Asociado al diseño universal surge el concepto de accesibilidad universal; como filosofía para todas las disciplinas del diseño del ambiente y como orientación que considere a las personas con sus diversas necesidades, determinadas ya sea por la etapa evolutiva en que se encuentran o bien por las habilidades y/o capacidades que posean (Huertas y Olivares, 2008). Este abarca cuatro ámbitos principales: la edificación, el urbanismo, el transporte y la comunicación considerando las interacciones entre los mismos y se plasma en la realidad del tejido urbano, facilitando la movilidad y el desenvolvimiento con seguridad en la ciudad.

Los conceptos de localización, accesibilidad y movilidad pueden ser considerados tres componentes fundamentales de la justicia o equidad espacial, concepto que se enmarca en un contexto mucho mayor en el que se considera que la esencia de la justicia social puede encarnarse en los criterios de necesidad, de contribución al bien común y de mérito (Drezner y Hamacher, 2002).

En este sentido, la sociedad del siglo XXI, en el llamado proceso de democratización de la gestión de las ciudades, reclama una visión más transversal de este ejercicio de pensar la ciudad; en la que el espacio público se convierta en elemento fundamental para garantizar justicia social y equidad urbana.

El lugar de encuentro, de interacción social, como articulador de la ciudad, el espacio público accesible es aquel que otorga el derecho a interactuar socialmente y a desarrollar las aptitudes y potencialidades en los diversos ámbitos de la actividad cotidiana, a utilizar y disfrutar libremente de todos los servicios que presta y ofrece la comunidad. (CONADIS, 2009).

Las pautas que determinan un diseño accesible y por ende que satisface las movilidades urbanas, la accesibilidad y la funcionalidad de los espacios públicos no se reducen a la eliminación de desniveles por medio de rampas y a la aplicación de un catálogo de dimensiones antropométricas adaptadas a un ser humano de capacidades diversas. Los análisis llevados a cabo para evaluar la accesibilidad deben partir de un enfoque sistémico que analice los problemas del espacio público urbano en conjunto y no de sus elementos componentes aislados. El acceso a un espacio público accesible debe ser considerado como un derecho de todo ser humano a tener la posibilidad de ingresar, transitar, permanecer y usar el lugar, de manera segura, confortable y autónoma, favoreciendo la interacción entre las personas como medida de la igualdad social. 


\section{Referencias Bibliográficas}

Borja, J. (1998) "Ciudadanía y espacio público.", Revista Ambiente y Desarrollo. 14(3), pp 13-22.

Borja, J. (2003) "La ciudad conquistada". Alianza Editorial. pp 29.

Brandao, P. (2011) "La imagen de la ciudad. Estrategias de identidad y comunicación". Barcelona: Publicacions i Edicions Universitat de Barcelona.

Capel, H. (2005) "El Modelo Barcelona: Un Examen Crítico". En: Barcelona: Ediciones del Serbal.

Cazanave, J. (2007) "Pautas metodológicas para el diseño de espacios de intercambio social accesible por adultos mayores". (Tesis de Doctorado. Facultad de Arquitectura, IPSJAE Ciudad de La Habana).

Coyula, M (2000) "El traje se empieza por el ojal." Revista Arquitectura y Urbanismo. En: ISPJAE (Ed). Habana. 21(3) pp 18-19.

Consejo Nacional de Discapacidades (CONADIS) (2009) "Guía de Accesibilidad al Medio Físico". CD- ROM. República de Ecuador.

Drezner, Z; Hamacher, H. (2002) "Facility location. Applications and Theory". SpringerVerlag, Alemania- EE UU.

Gehl, J; Gemzoe, L. (2001) "New City Spaces." Copenhague. The Danish Architectural Press. (www.freebookspot.com)

Herce M. (2000) "Barcelona: Accesibility changes and metropolitan transformation", built environment. Oxford: Alexandrine Press.

Huertas, M; Olivares-Melero, JM. (2008) "Taller de Barreras Arquitectónicas y Urbanísticas". "curso de Voluntariado".

Jacobs, J. (1992) "The Death and Life of Great American Cities". EE UU NY ed.

Lefebvre, H. (1974) "The production of space". Oxford: Basil.

Organización Mundial de la Salud (OMS) (2001) "Clasificación Internacional de Funcionamiento Discapacidad y Salud". En: OMS (Ed), CD-ROM; mutilingüe, versión 1.0, Ginebra.

Saldarriaga, A. (2002) "La Arquitectura como Experiencia; Espacio; Cuerpo y Sensibilidad". En Villegas (ed). Universidad Nacional de Colombia. pp 211.

Talen, E. (2001): "Acces: Geographical". En: International Encynclopedia of the Social and Behavioral Sciences. Elsevier, EE UU, Vol A-B. pp 30-33.

Ventura, F. (2012) "Miradas Excéntricas. Genealogías Forzadas, Sumideros Urbanos y Ciudades Extremas". En: Recolectores Urbanos (Ed). Sevilla. pp 27. 